\title{
Transient Behavior of Space Charge in Heat-Treated Low-Density Polyethylene under Coupled Fields
}

\author{
Ling Zhang, ${ }^{1,2}$ Yuanxiang Zhou, ${ }^{1 *}$ Chenyuan Teng, ${ }^{3}$ Yunxiao Zhang, ${ }^{1}$ and Zixia Cheng ${ }^{3}$ \\ ${ }^{1}$ Department of Electrical Engineering, Tsinghua University, Beijing 100084, China \\ ${ }^{2}$ State Key Laboratory of Electrical Insulation and Power Equipment, Xi' an Jiaotong University, \\ Xi'an, Shaanxi 710049, China \\ ${ }^{3}$ School of Electrical Engineering, Zhengzhou University, Zhengzhou 450001, China
}

(Received December 6, 2016; accepted June 16, 2017)

Keywords: low-density polyethylene, space charge, coupled field, negative differential mobility (NDM), trap-level distribution

Developing high-performance polyethylene-based insulating materials for high-voltage direct current (HVDC) application is still a great challenge owing to space charge effects especially under DC conditions. In particular, microscopic crystal-amorphous structures make the charge transport process more complex under coupled fields, i.e., electric, temperature, and mechanical fields. Here, we investigate the effect of heat treatment on the morphology and space charge behavior of low-density polyethylene (LDPE) under temperature fields ranging from 20 to 60 ${ }^{\circ} \mathrm{C}$ under a high external DC field of $-100 \mathrm{kV} / \mathrm{mm}$. The results show that better crystallization in LDPE was achieved with a lower annealing rate, and both the size and level of uniformity of spherulites increased. Positive and negative charge packets were observed and followed the fielddependent negative differential mobility (NDM) law, which were strongly affected by temperature. According to the change in trap property, the effect of crystal-amorphous regions is critical to long-range hole transport, because larger spherulites showed increased difficulty on interchain tunneling, reduced carrier mobility, and reinforced hole trapping.

\section{Introduction}

Currently, polyethylene-based insulating materials have shown great potential for applications in high-voltage direct current (HVDC) apparatuses owing to their excellent insulation properties, i.e., high resistivity and low carrier mobility. ${ }^{(1)}$

Polyethylene is a semicrystalline polymer, whose morphology and structure are strongly associated with heat treatment and additives. ${ }^{(2,3)}$ Spherulites in the crystalline region of polyethylene consist of chain-folded lamellae, which are usually molded through hot melting and annealing. Nonisothermal annealing has a considerable effect on the crystallinity of polymers: slower annealing results in larger spherulites and higher crystallinity. ${ }^{(4,5)}$ Hot melting and annealing processes are used in the actual processing and manufacture of extruded power cables as well. Thus, investigation into the relationship between the nonisothermal crystallization and the morphological structure is not only of great academic value, but also significant to engineering practice.

"Corresponding author: e-mail: zhou-yx@tsinghua.edu.cn http://dx.doi.org/10.18494/SAM.2017.1542 
As a main candidate for HVDC power cable insulation, polyethylene-based insulation suffers from coupled fields in a practical service environment wherein temperature makes charge evolution more complicated: conductivity increases nonlinearly with temperature, and the temperature gradient results in the heterogeneous distribution of conductivity and severe field distortion. ${ }^{(6)}$ Thus, the law and mechanism of nonisothermal crystallization in relation to the transient behavior of space charge and external current are of significance in polyethylene.

The aim of this work is to investigate the transient behavior of space charge and external current in heat-treated low-density polyethylene (LDPE) under coupled fields. For this purpose, a ms-level simultaneous measurement system of space charge and external current was developed (Fig. 1). The main results, consisting of quantitative analysis of charge injection, field distortion, space charge packet behavior, and so forth, are discussed here.

\section{Materials and Methods}

\subsection{Materials and sample preparation}

LDPE pellets were purchased from Sigma-Aldrich (CAS 9002-88-4) with the melt index of $25 \mathrm{~g} / 10 \mathrm{~min}$, density of $0.925 \mathrm{~g} / \mathrm{ml}\left(25^{\circ} \mathrm{C}\right)$, and melt point of $116^{\circ} \mathrm{C}$. LDPE films $(190 \pm 10 \mu \mathrm{m})$ were prepared via hot pressing using polyimide films as substrates at $140{ }^{\circ} \mathrm{C}$ and $10 \mathrm{MPa}$ for $5 \mathrm{~min}$ pressing. After the hot pressing, the LDPE films experienced three typical annealing processes, i.e., ice water (I), air (A), and slow (S) annealing. The resultant samples were denoted as LD-I, LD-A, and LD-S, respectively. Note that the slow annealing process was carried out by holding the samples in the molds without pressure. To avoid the effect of moisture, all samples were subsequently vacuumed at $70{ }^{\circ} \mathrm{C}$ overnight and then stored in a drier.

\subsection{Characterization of crystalline structure}

The effect of annealing rate on the crystallinity and melt point of the LDPE samples during the nonisothermal crystallization was determined by Q2000 Differential Scanning Calorimetry (DSC). The temperature range was from 20 to $180^{\circ} \mathrm{C}$ at a ramp rate of $10^{\circ} \mathrm{C} / \mathrm{min}$ under a $\mathrm{N}_{2}$ flow. The heating curve of the first round was used to compare the effect of annealing history on the crystalline morphology.

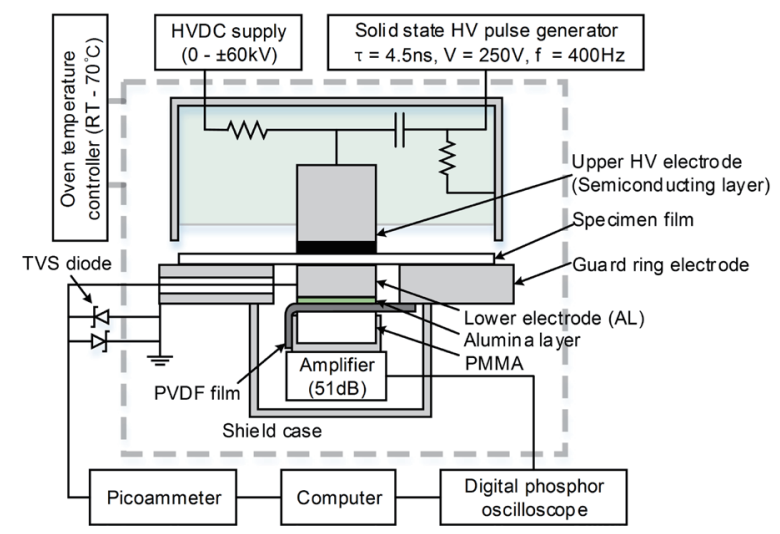

Fig. 1. (Color online) Schematic diagram of ms-level system for simultaneous measurement of space charge and external current. 
In order to observe the microscopic cell morphology of the heat-treated LDPE samples, $\sim 10 \mu \mathrm{m}$ films were prepared between two glass slides, and a polarizing microscope (POM) was utilized with the transmission mode and 500-fold magnification.

\subsection{Thermally stimulated current measurement}

The effect of heat treatment on the trap level property was investigated by the thermally stimulated current (TSC) method. ${ }^{(7)}$ Film samples were coated with gold electrodes and polarized under DC $30 \mathrm{kV} / \mathrm{mm}$ at $50{ }^{\circ} \mathrm{C}$ for 20 min under a $\mathrm{N}_{2}$ atmosphere to avoid moisture. Then, the TSC cell was rapidly cooled to $-70{ }^{\circ} \mathrm{C}$ and shorted for 3 min after the high voltage was turned off. The TSC was recorded via a Keithley6485 picoammeter while the temperature was linearly increased at a ramp rate of $5{ }^{\circ} \mathrm{C} / \mathrm{min}$. Trap depth and corresponding trap density were calculated by the method reported in detail previously. ${ }^{(8)}$

\subsection{Simultaneous measurement of space charge and external current}

An advanced ms-level simultaneous measurement system of space charge and external current was developed on the basis of the pulsed electroacoustic (PEA) method and the threeelectrode method, respectively. ${ }^{(9)}$ A $9 \mu \mathrm{m} \beta$-polyvinylidene fluoride (PVDF) film was used as the piezoelectric sensor, which possesses a wide operating frequency $(0-500 \mathrm{MHz})$. The voltage endurance capability of the PEA unit could reach $\pm 60 \mathrm{kV}$ DC with a new design of high-voltage electrodes. Temperature was conditioned via an oven up to $70{ }^{\circ} \mathrm{C}$. The main parameters of the solid-state high-voltage pulse generator were $1 \mathrm{kHz}, 250 \mathrm{~V}$, and $4.5 \mathrm{~ns}$.

In this work, the external DC field was chosen as $-100 \mathrm{kV} / \mathrm{mm}$, resulting in a good signalto-noise ratio of the PEA system. Thus, the average number of filters for PEA signals could be reduced from hundreds or thousands to just 10 or even less, for the subsequent data processing. By utilizing a large-capacity storage module of $500 \mathrm{MB}$ for the digital oscilloscope, PEA measurements with a high-speed acquisition of $1 \mathrm{~ms}$ could be continuously performed for as long as $10 \mathrm{~min}$, which was denoted as high-speed mode. For the steady measurement, e.g., space charge evolution at room temperature, the oscilloscope was set in average acquisition with an interval of $5 \mathrm{~s}$, which was denoted as low-speed mode. Furthermore, the external current and space charge could be recorded simultaneously. A thin alumina $\left(\mathrm{Al}_{2} \mathrm{O}_{3}\right)$ layer was used for the signal separation owing to its insulation property and similar acoustic impedance to aluminum electrodes.

The transient behavior of space charge was measured using the high-speed mode of the PEA system at 40 and $60{ }^{\circ} \mathrm{C}$ for $3 \mathrm{~min}$, and the interval of $5 \mathrm{~s}$ was chosen using the low-speed mode at $20^{\circ} \mathrm{C}$ for $60 \mathrm{~min}$. Each group was tested at least twice.

\section{Results}

\subsection{Morphological structure of LDPE samples}

Figure 2 shows the typical DSC heating curves of the first round of heat-treated LDPE samples. It is clear that the effect of annealing on the melting point ranging from 111.5 to $112.0{ }^{\circ} \mathrm{C}$ is almost negligible. However, what attracts special attention was that the peak width at half height 


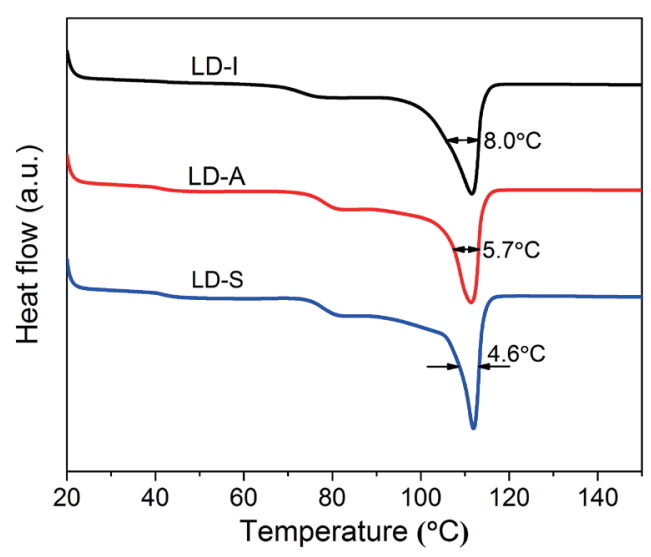

Fig. 2. (Color online) DSC heating curves of LDPE samples.

decreased with a smaller annealing rate, which might be related to the parameters of crystalline cells including size and uniformity. On the basis of DSC curves, the crystallinity was calculated and the results, i.e., $36 \%$ for LD-I, 39\% for LD-A, and $40 \%$ for LD-S, indicate that a slow annealing rate benefits the crystalline process. ${ }^{(10)}$

Figure 3 shows the POM observations of cell microscopic morphology. The bright part indicates the crystal regions, and the dark part probably is the amorphous regions and crystalamorphous interfaces. Obviously, both the size and uniformity increased with a slower annealing rate. Specifically, $\sim 20 \mu \mathrm{m}$ cells homogeneously appeared in LD-S samples owing to the complete growth of the crystals, and 2-5 $\mu \mathrm{m}$ cells distributed in LD-A samples with a higher density. In comparison, a wider size distribution was found in LD-I samples, ranging from 0.5 to $2.69 \mu \mathrm{m}$.

\subsection{Space charge and external current density at RT}

Under a certain high DC field, it is usual to observe an interesting phenomenon known as a space charge packet as a pulse of net charge that remains in the form of a pulse as it transits across the insulation. ${ }^{(11,12)}$

Figure 4 shows the external current, maximum distortion field, and space charge dynamics in different heat-treated LDPE samples under $-100 \mathrm{kV} / \mathrm{mm}$ at RT. In Figs. 4(a)-4(c), a positive space charge packet near the anode appeared in all the samples and migrated toward the cathode. The space charge packet moved fastest in LD-I and slowest in LD-S, which has the maximum amount of injected charges.

The moving space charge packet strengthened its front electrical field while weakening the rear, and the neutralization of positive and negative space charge packets after the encounter also enhanced the electrical field in the bulk. Therefore, the inner maximum distortion fields of three samples in Figs. 4(d)-4(f) increase as the frontiers of positive and negative space charge packets become closer, among which, the value of LD-A is the largest owing to its maximum amount of negative space charge encountering with the positive space charge packet.

The synchronous external current measurement results of heat-treated LDPE samples in Figs. 4(g)-4(i) show that the conductivity decreases with annealing rate. The higher annealing rate results in the smaller, more irregular, and more nonuniform spherulite size, which might increase the size of the crystal-amorphous interfacial regions. 


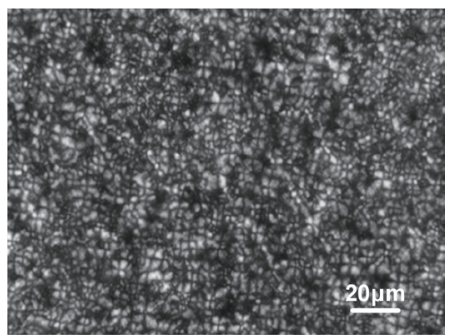

(a)

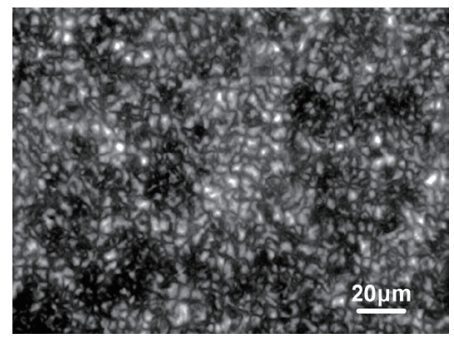

(b)

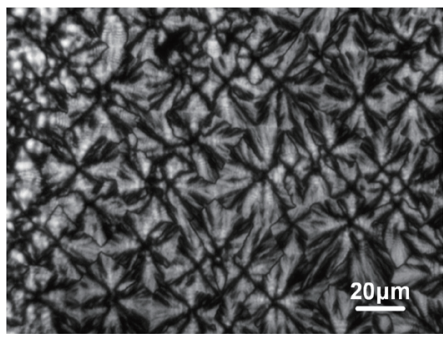

(c)

Fig. 3. Cell morphologies of LDPE samples: (a) LD-I, (b) LD-A, and (c) LD-S.

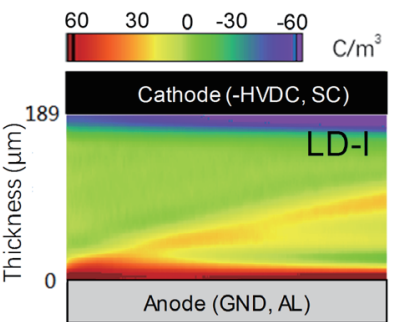

(a)

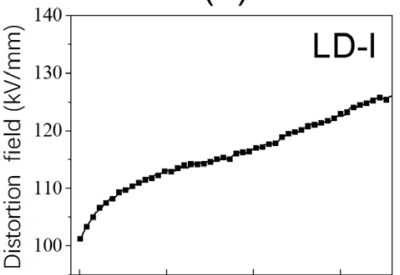

(d)

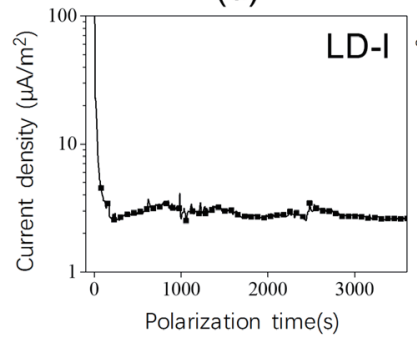

(g)

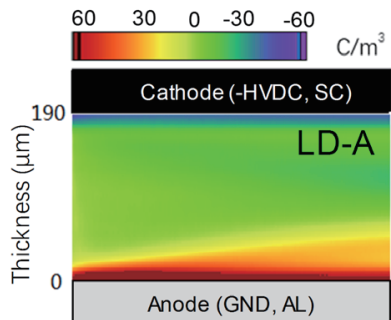

(b)

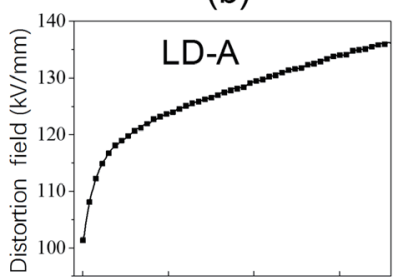

(e)

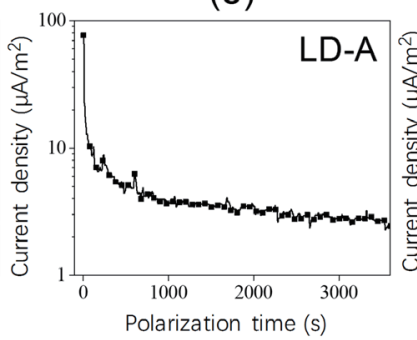

(h)

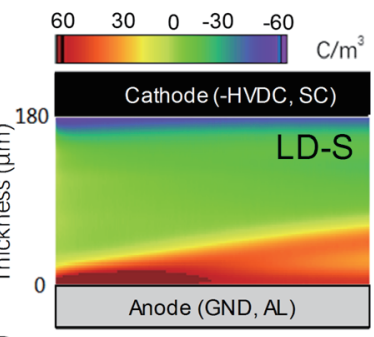

(c)

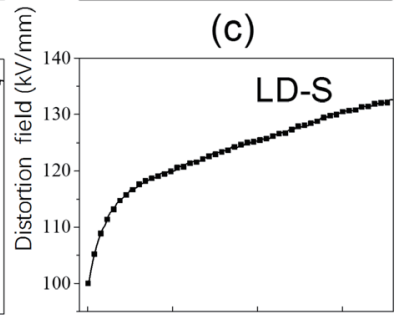

(f)

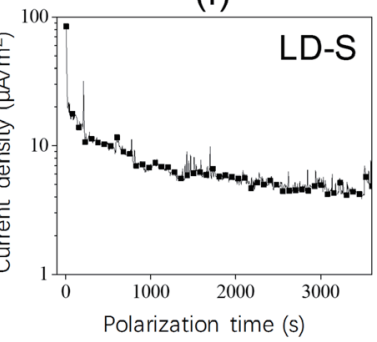

(i)

Fig. 4. (Color online) Relationship of (a)-(c) space charge and (d)-(f) maximum distortion field with (g)-(i) external current density in heat-treated LDPE within $60 \mathrm{~min}$ under $-100 \mathrm{kV} / \mathrm{mm}$.

\subsection{Transient space charge at $60^{\circ} \mathrm{C}$}

Figure 5 illustrates the transient evolution of space charge in heat-treated LDPE at $60{ }^{\circ} \mathrm{C}$ under $-100 \mathrm{kV} / \mathrm{mm}$, which is divided into three different time scales, namely, $0-1 \mathrm{~s}, 0-10 \mathrm{~s}$, and $0-1$ min. Space charge packets in all three groups appeared rapidly within $1 \mathrm{~s}$ when the temperature is increased to $60^{\circ} \mathrm{C}$. The polarity of space charge packets is positive in LD-I, negative in LD-S, and both positive and negative in LD-A after $1 \mathrm{~s}$ polarization. The main space charge that remained is positive in LD-I, negative in LD-A, and negative in LD-S $10 \mathrm{~s}$ later. It is also seen in Figs. 4(c), 4(f), and 4(i) that only in LD-A and more than one space charge packet appear during the $1 \mathrm{~min}$ polarization. 


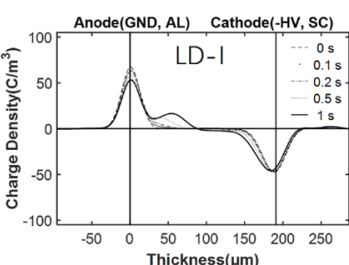

(a)

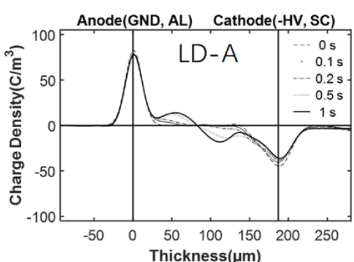

(d)

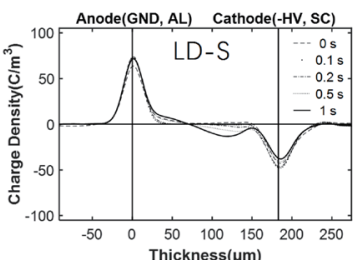

(g)

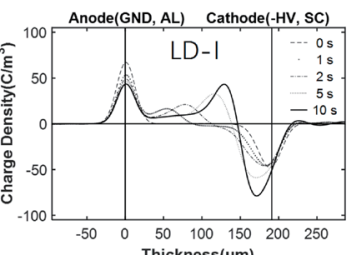

(b)

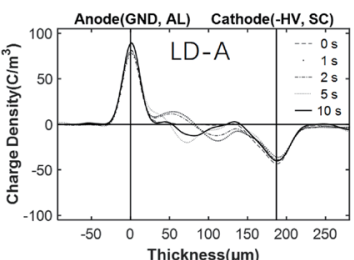

(e)

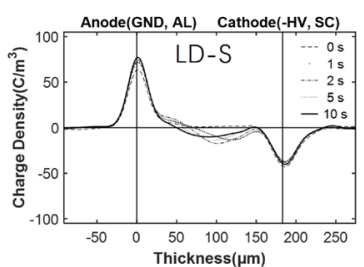

(h)

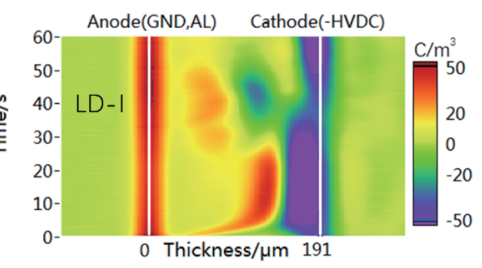

(c)

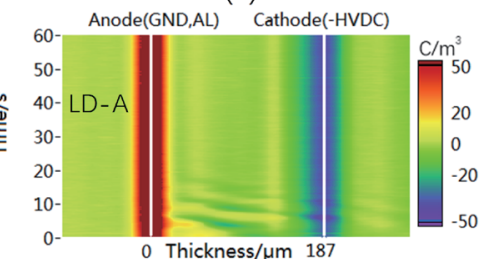

(f)

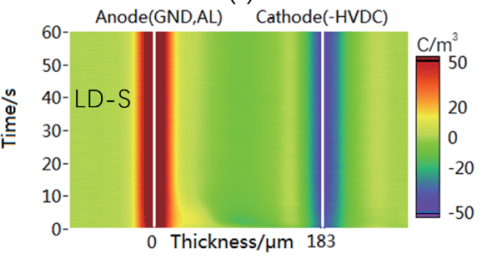

(i)

Fig. 5. (Color online) Transient evolution of space charge in heat-treated LDPE at $60{ }^{\circ} \mathrm{C}$ under $-100 \mathrm{kV} / \mathrm{mm}$ : (a)-(c) LD-I, (d)-(e) LD-A, and (g)-(i) LD-S.

\section{Discussion}

\subsection{Effect of temperature on dynamic charge evolution}

In order to depict in more detail the features of charge packets, the peak positions of positive charge packets are plotted as they transit the insulation. The decrease in the velocity of the peak is similar at each temperature field. Moreover, the migration velocity and the sequence of the formation of charge packets follow this order: 60,40 , and $20^{\circ} \mathrm{C}$. Note that space charge packets do not always arrive and dissipate in the electrodes, which is closely associated with the heterocharge packet in the bulk (Fig. 6).

\subsection{Effect of annealing rate on charge injection and transport}

Figure 7 shows a model of injection and transport of space charge packets from the electrode. The surface charge on the electrode could be regarded as a thickness-negligible charge layer [see the bold black line in Fig. 7(a)] as well as the space charge packets. However, the obtained PEA signal has a quasi-Gaussian shape owing to the system function, including the high-voltage pulse shape, the thickness of the PVDF sensor, and the effects of attenuation and dispersion on pressurewave propagation.

Charge injection immediately starts through the electrode/polymer interface via the Schottky effect when a high electric field is applied. During the transit of the charge packet, the field after it continuously relaxes, leading to the increase in the Schottky injection barrier height and the 


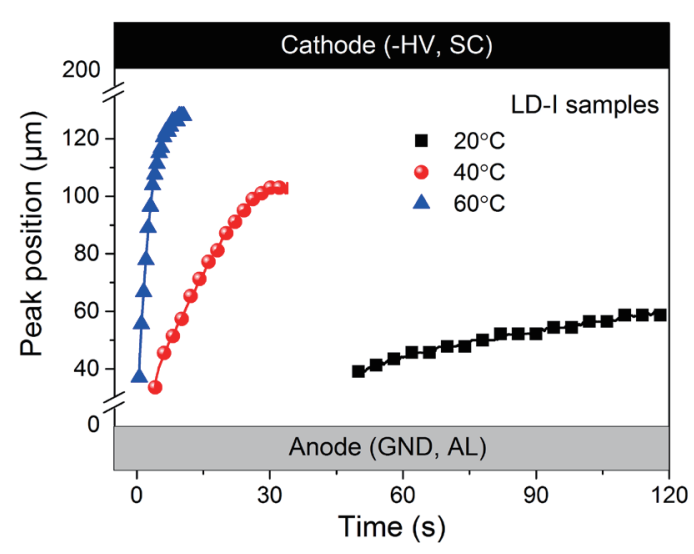

Fig. 6. (Color online) Relationship between packet peak position and polarization time under $-100 \mathrm{kV} / \mathrm{mm}$ at different temperatures.

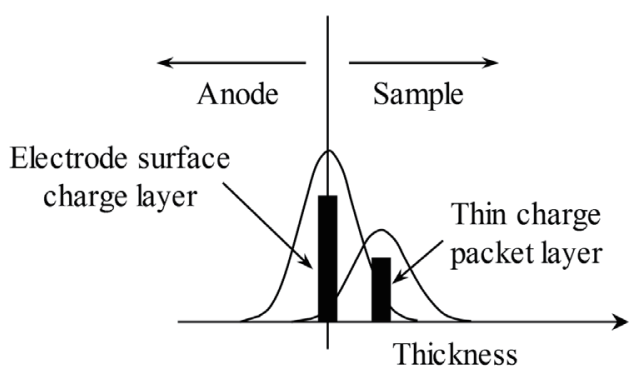

(a)

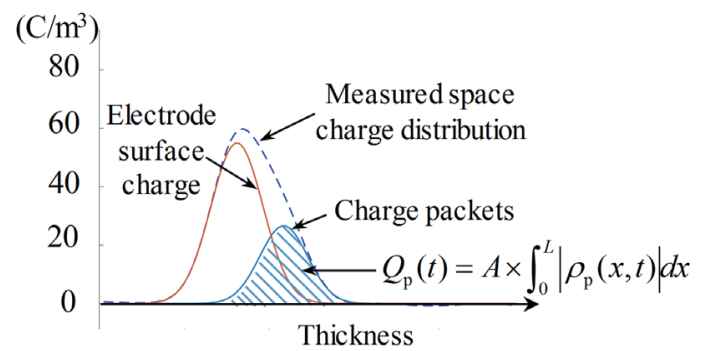

(b)

Fig. 7. (Color online) Calculation method of the total amount of space charge packet: (a) schematic diagram and (b) measured data and peak-separation results.

formation of a blocking effect for the subsequent charge injection. Then, the packet continues to move towards the other electrode following the field-dependent negative differential mobility (NDM) law until recombination and extraction. ${ }^{(13,14)}$ Figure $7(\mathrm{~b})$ shows the measured space charge profile and peak-separation results involving the polarization process within $1 \mathrm{~s}$ at $60{ }^{\circ} \mathrm{C}$ under $-100 \mathrm{kV} /$ $\mathrm{mm}$. The injection amount of the charge packet can be integrated as

$$
Q_{P}(t)=A x \int_{0}^{L}\left|\rho_{P}(x, t)\right| d x
$$

where $Q_{P}(t)$ is the injected amount of space charge (C) at time $t(\mathrm{~s})$, A is the surface area $\left(\mathrm{m}^{2}\right)$ of the upper electrode of the PEA unit, $L$ is the thickness $(\mathrm{m})$ of the samples, and $\rho_{P}(x, t)$ is the charge concentration $\left(\mathrm{C} / \mathrm{m}^{3}\right)$ at time $t$ and location $x$.

When the injected charge packet has not been isolated from the electrode, the waveforms of the charge packet and the electrode surface charge layer partially overlap. A multi-peak separation technique is utilized to obtain the individual charge packet profile, which is shadowed in Fig. 7(b).

The charge injection process is of critical significance for the subsequent charge dynamic behavior in the bulk of polymers. Figure 8 shows the total injected amount of charge packets in heat-treated LDPE samples within $10 \mathrm{~s}$ under $-100 \mathrm{kV} / \mathrm{mm}$ at $60{ }^{\circ} \mathrm{C}$ using the model and method 


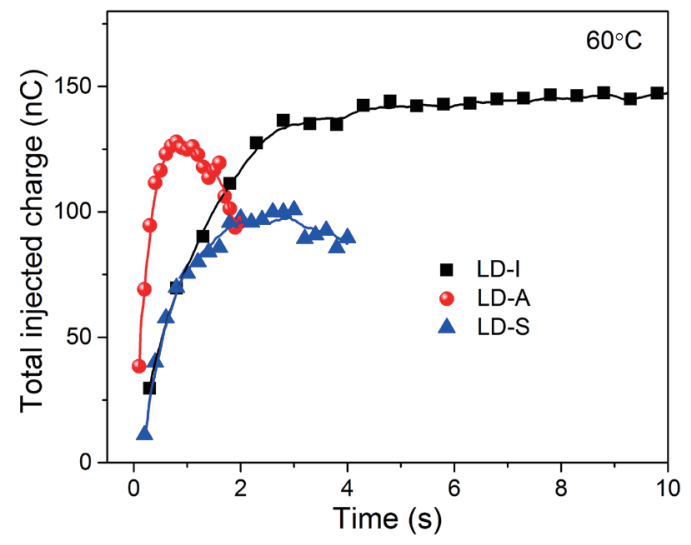

Fig. 8. (Color online) Relationship between the total amount of injected charges and the annealing rate under $-100 \mathrm{kV} / \mathrm{mm}$ at $60^{\circ} \mathrm{C}$.

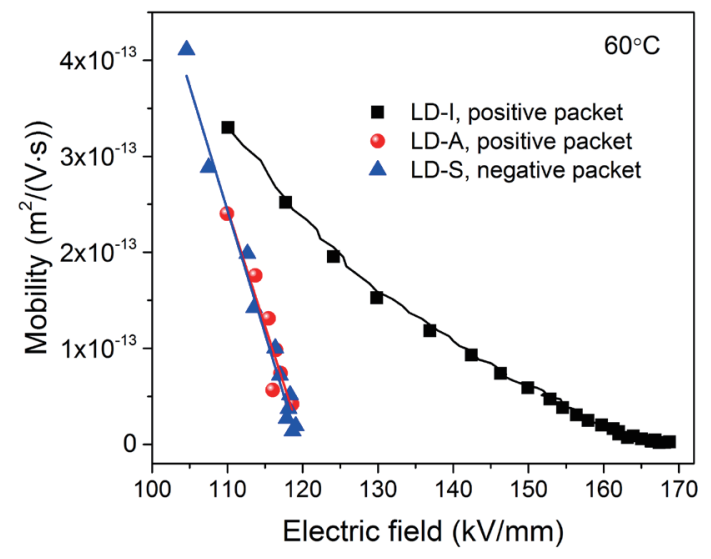

Fig. 9. (Color online) Field-dependent negative differential mobility in heat-treated LDPE under -100 $\mathrm{kV} / \mathrm{mm}$ at $60{ }^{\circ} \mathrm{C}$.

described in Fig. 7. All the samples show a rapid increase in the total amount of injected charges at the initial stage within $1 \mathrm{~s}$, and LD-A samples have the largest amount of injected charges owing to the appearance of both positive and negative charge packets at the same time [see Fig. 5(d)]. In the comparison of LD-I and LD-S, the former has a positive charge packet [see Fig. 5(a)] and the latter has a negative one [see Fig. 5(g)], but the injection rates were almost equal at the first second. Moreover, both LD-A and LD-S encountered a decrease in the charge amount after the meet, recombination, and extraction processes. In contrast, the amount of positive charge packet in LD-I seemed to be saturated after $2 \mathrm{~s}$ owing to the slow movement and accumulation of negative charges in the vicinity of the cathode.

In order to analyze the propagation of the charge packets, the mobility of the charge packet obtained from the space charge profiles was presented under $-100 \mathrm{kV} / \mathrm{mm}$ at $60{ }^{\circ} \mathrm{C}$ (Fig. 9). One remarkable feature is that the mobility of the positive packet in LD-A and that of the negative packet in LD-S overlap each other and radically decrease to zero at $\sim 120 \mathrm{kV} / \mathrm{mm}$ following the NDM law. ${ }^{(15)}$

\subsection{Morphology and related trap level in heat-treated LDPE}

Figure 10 shows the trap-level distribution of heat-treated LDPE samples. The results indicate that the peaks of the trap levels are in the range of $0.95-1.00 \mathrm{eV}$, which are assumed to be deep trapping sites and agree well with the previous results. ${ }^{(16,17)}$ The peak trap densities of LD-I, LDA, and LD-S are $5.3 \times 10^{20}, 4.0 \times 10^{20}$, and $4.2 \times 10^{20} /\left(\mathrm{eV} \cdot \mathrm{m}^{3}\right)$, respectively. It is also shown that there are much fewer trapping sites in LD-S than in the other two groups, which might be related to the uniform cell size and the smaller crystal-amorphous interfacial regions. However, it is contradictory that LD-I samples have the largest carrier mobility (see Fig. 9) and the highest trap density of deep trapping sites at the same time based on the trap level distribution. As a typically simple semicrystalline polymer, LDPE consists of spherulites formed from nucleating sites with 


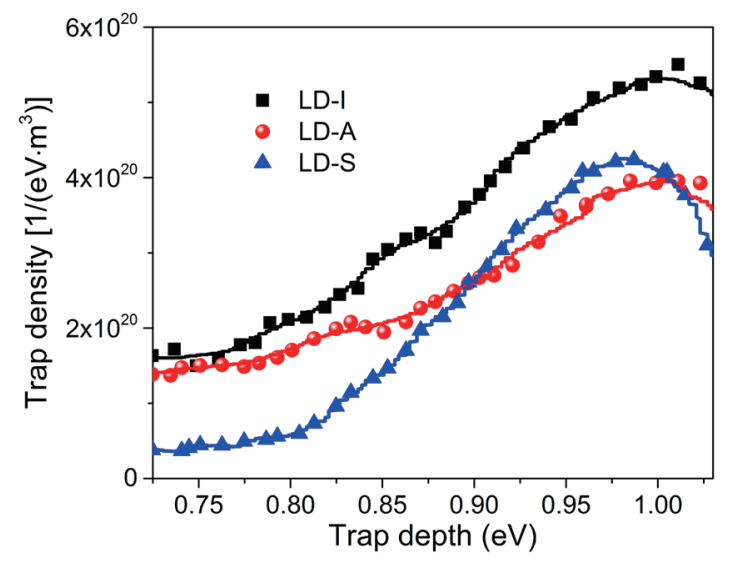

Fig. 10. (Color online) Trap level distribution of heat-treated LDPE samples.

diameters of micrometer level. Most of the deep trapping sites $(\sim 0.92 \mathrm{eV}$ in Ref. 7) formed from physical defects occurred in the crystal-amorphous interfacial regions, which mainly consist of chain branches, chain ends, short polymer chains, impurities, and so forth, that strongly affect the charge carrier transport process. ${ }^{(18,19)}$

Here, we propose a mechanism that could explain the effect of heat treatment on the charge carrier transport shown in Fig. 5. Although the annealing rate did alter the trap level distribution, the extent was relatively limited compared with the radical change in the microscopic morphology of the LDPE samples. In contrast to electron transport, hole transport can take place while confined to polymer chains, and long-range hole conduction requires interchain hole tunneling. ${ }^{(20)}$ Thus, hole transport is more sensitive to morphology conformations in the amorphous regions. As a consequence, the difficulty of finding available paths across the spherulites in the field direction rapidly increases with the increase in spherulite size, resulting in the reduced hole mobility and encouraged hole trapping.

\section{Conclusions}

In this study, an advanced ms-level PEA system was developed for the measurement of the transient behavior of space charge in heat-treated LDPE samples under coupled fields. From the DSC curves and POM images, it could be concluded that a slower annealing rate contributes to the complete crystallization of LDPE with larger spherulites and better uniformity. Both positive and negative charge packets followed the field-dependent NDM law, and the transit and mobility of positive charge packets were radically affected by the temperature fields. In comparison with the limited change in trap level distribution, the morphology conformation of spherulites played a more dominant role in affecting long-range hole transport by decreasing the hole mobility and increasing the hole trapping. 


\section{Acknowledgments}

We are grateful for the financial support of the State Key Development Program of Basic Research of China (973 Program) (2014CB239501), National Natural Science Foundation of China (50377089), State Key Laboratory of Electrical Insulation and Power Equipment (EIPE16208), project of JIBEI Electric Power Company Research Institute of State Grid (52018K15000R), and China Postdoctoral Science Foundation (2016M591176).

\section{References}

1 T. L. Hanley, R. P. Burford, R. J. Fleming, and K. W. Barber: IEEE Electr. Insul. Mag. 19 (2003) 13.

2 Y. L. Chong, G. Chen, I. L. Hosier, A. S. Vaughan, and Y. F. F. Ho: IEEE Trans. Dielectr. Electr. Insul. 12 (2005) 1209.

3 J. X. Li, Y. W. Zhang, F. H. Zhang, and C. S. Wu: Jpn. J. Appl. Phys. 12 (2004) 8130.

4 Y. X. Zhou, N. H. Wang, P. Yan, X. D. Liang, and Z. C. Guan: J. Electrostat. 57 (2003) 381.

5 Y. X. Zhou, Y. S. Wang, M. Zahn, N. H. Wang, Q. H. Sun, X. D. Liang, and Z. C. Guan: Jpn. J. Appl. Phys. 50 (2011) 017101.

6 D. Fabiani, G. C. Montanari, C. Laurent, G. Teyssedre, P. H. F. Morshuis, R. Bodega, and L. A. Dissado: IEEE Electr. Insul. Mag. 24 (2008) 5.

7 M. Ieda: IEEE Trans. Electr. Insul. 19 (1984) 162.

8 F. Q. Tian, W. B. Bu, L. S. Shi, C. Yang, Y. Wang, and Q. Q. Lei: J. Electrostat. 69 (2011) 7.

9 Y. Li, M. Yasuda, and T. Takada: IEEE Trans. Dielectr. Electr. Insul. 1 (1994) 188.

10 O. Almanza, M. A. Rodriguez-Perez, B. Chernev, J. A. De Saja, and P. Zipper: Eur. Polym. J. 41 (2005) 599.

11 A. See, L. A. Dissado, and J. C. Fothergill: IEEE Trans. Dielectr. Electr. Insul. 8 (2001) 859.

12 N. Hozumi, T. Takeda, H. Suzuki, and T. Okamoto: IEEE Trans. Dielectr. Electr. Insul. 5 (1998) 82.

13 J. F. Xia, Y. W. Zhang, F. H. Zheng, Z. L. An, and Q. Q. Lei: J. Appl. Phys. 109 (2011) 034101.

14 L. A. Dissado: Proc. IEEE Int. Conf. Solid Dielectr. ICSD2010 (IEEE, 2010) p. 1.

15 G. Teyssedre, T. T. N. Vu, and C. Laurent: Appl. Phys. Lett. 107 (2015) 252901.

16 F. Q. Tian, Q. Q. Lei, X. Wang, and Y. Wang: Appl. Phys. Lett. 99 (2011) 142903.

17 B. Han, X. Wang, Z. Sun, J. M. Yang, and Q. Q. Lei: Appl. Phys. Lett. 102 (2013) 012902.

18 J. P. Jones, J. P. Llewellyn, and T. J. Lewis: IEEE Trans. Dielectr. Electr. Insul. 12 (2005) 951.

19 W. W. Shen, H. B. Mu, G. J. Zhang, J. B. Deng, and D. M. Tu: J. Appl. Phys. 113 (2013) 083706.

20 S. Serra, E. Tosatti, S. Iarlori, S. Scandolo, G. Santoro, and M. Albertini: IEEE Conf. Electr. Insul. and Dielectr. Phenom. (IEEE, 1998) p. 19.

\section{About the Authors}

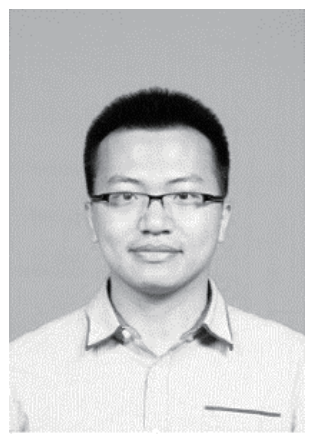

Ling Zhang was born in Fujian Province, China, in 1987. He received his B.Sc. degree in 2010 and Ph.D. degree in Electrical Engineering in 2015 from Tsinghua University, China. He is now a postdoctoral researcher in the Institute of High Voltage and Insulation Technology in Tsinghua University. His research interests include nanoparticle-polymer interface, nanodielectrics, advanced PEA technology, and space charge phenomena in solid insulating materials. 


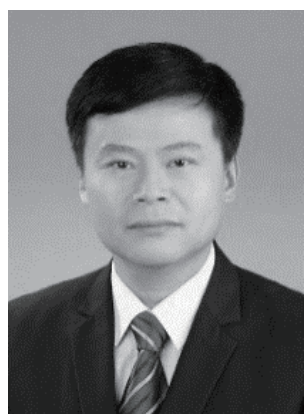

Yuanxiang Zhou (M'04) was born in Fujian Province, China in 1966. He received his B.S. degree from Tsinghua University, China in 1988, M.Sc. degree from the Electrical Power Research Institute, China in 1991, and Ph.D. degree from Akita University, Japan in 1999. From 1999 to 2000, he did research in NIRE, AIST, Japan as a NEDO fellow. At present, he is a professor of Tsinghua University and IET fellow. His interests include organic and inorganic dielectrics, high-voltage and insulation technology, electrical equipment, and onsite detection and diagnosis.

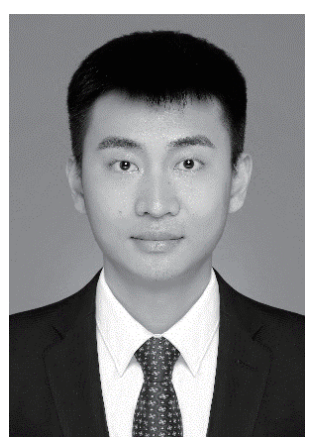

Chenyuan Teng was born in Zhejiang Province, China, in 1992. He received his B.Sc. degree in 2014 from North China University of Water Resources and Electric Power, Zhengzhou, China. He is now a graduate student in the School of Electrical Engineering in Zhengzhou University. His research field is space charge characteristics of polyethylene-based insulating materials.

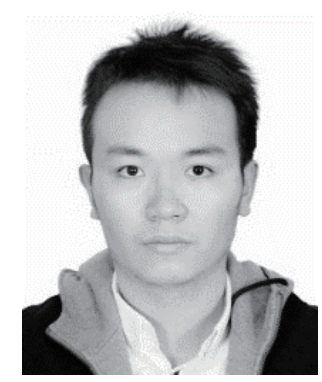

Yunxiao Zhang was born in Fujian Province, China in 1990. He received his B.Sc. degree in 2013 from Tsinghua University, China. He is now a Ph.D. candidate in the State Key Lab of Control and Simulation of Power Systems and Generation Equipment of the Department of Electrical Engineering in Tsinghua University. His research fields are electrical treeing phenomenon in insulating materials and cable design.

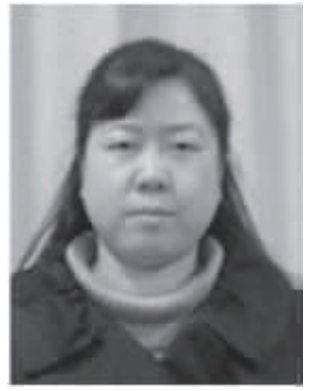

Zixia Cheng was born in Henan Province, China, in 1974. She received her B.Sc. and M.Sc. degrees from Harbin University of Science and Technology, Harbin, China, in 1997 and 2000, respectively. She obtained her Ph.D. degree from Tsinghua University, Beijing, China, in 2005. Currently, she is a lecturer of the School of Electrical Engineering in Zhengzhou University. Her interests include electrical treeing and space charge phenomena in dielectrics, high-voltage technology, and analysis of electric power systems. 